\title{
Barium Stars: Theoretical Interpretation
}

\author{
Laura Husti $^{\mathrm{A}, \mathrm{B}, \mathrm{D}}$, Roberto Gallino $^{\mathrm{A}}$, Sara Bisterzo ${ }^{\mathrm{A}}$, Oscar Straniero ${ }^{\mathrm{C}}$, \\ and Sergio Cristallo ${ }^{\mathrm{C}}$ \\ A Dipartimento di Fisica Generale, Università degli Studi di Torino, via P. Giuria 1, \\ 10125 Torino, Italia \\ ${ }^{B}$ Research Centre for Atomic Physics and Astrophysics, University of Bucharest, \\ P.O. Box MG-6, RO-077125 Bucharest-Magurele, Romania \\ ${ }^{\mathrm{C}}$ INAF Osservatorio Astronomico di Collurania, via M. Maggini, 64100 Teramo, Italy \\ D Corresponding author. Email: lotesileanu@yahoo.com
}

Received 2008 December 16, accepted 2009 August 7

\begin{abstract}
Barium stars are extrinsic Asymptotic Giant Branch (AGB) stars. They present the $s$-enhancement characteristic for AGB and post-AGB stars, but are in an earlier evolutionary stage (main sequence dwarfs, subgiants, red giants). They are believed to form in binary systems, where a more massive companion evolved faster, produced the $s$-elements during its AGB phase, polluted the present barium star through stellar winds and became a white dwarf. The samples of barium stars of Allen \& Barbuy (2006) and of Smiljanic et al. (2007) are analysed here. Spectra of both samples were obtained at high-resolution and high S/N. We compare these observations with AGB nucleosynthesis models using different initial masses and a spread of ${ }^{13} \mathrm{C}$-pocket efficiencies. Once a consistent solution is found for the whole elemental distribution of abundances, a proper dilution factor is applied. This dilution is explained by the fact that the $s$-rich material transferred from the AGB to the nowadays observed stars is mixed with the envelope of the accretor. We also analyse the mass transfer process, and obtain the wind velocity for giants and subgiants with known orbital period. We find evidence that thermohaline mixing is acting inside main sequence dwarfs and we present a method for estimating its depth.
\end{abstract}

Keywords: stars: abundances — stars: AGB and post-AGB — (stars:) binaries: general — stars: chemically peculiar — stars: mass loss

\section{Introduction}

Barium stars have been a fascinating subject since their discovery by Bidelman \& Keenan (1951). Understanding their peculiar abundance patterns has been a challenge for over 50 years.

Barium stars show $s$-process enhancement. However, these stars are giants or main-sequence, far away from the thermal pulse asymptotic giant branch stars (TP-AGB) phase, where the $s$-process is manufactured. Late on the TP phase, AGB stars undergo recurrent third dredge-up (TDU) episodes, mixing with the envelope C-rich and $s$-process-rich material synthesised in the $\mathrm{He}$ intershell (the zone between $\mathrm{H}$ shell and $\mathrm{He}$ shell). The most likely interpretation of barium stars is that they acquired the $s$ enrichment by mass transfer in a binary system through stellar winds from an AGB companion that became later on a white dwarf (in some cases detectable, see Boffin \& Jorissen 1988, Jorissen \& Mayor 1988 and references therein). The study of barium stars is important for a better understanding of the $s$-process in AGB stars, of the mass transfer in binary systems and of the mixing processes, like thermohaline mixing, which may occur in the envelope of main sequence stars (Stancliffe et al. 2007 and references therein). This is a process that occurs when the mean molecular weight of the stellar material increases towards the surface. The heavier material transferred from the AGB will sink until the difference in molecular gradient weight will disappear.

A recent homogeneous sample of 26 barium stars with lead measurement detected at high-resolution spectroscopy ( $\mathrm{S} / \mathrm{N} \sim 100$ to 250 ) has been analysed by Allen \& Barbuy (2006). About half of these stars are barium dwarfs, and half are barium subgiants and giants. Many heavy elements have been observed, among which, Sr, $\mathrm{Y}, \mathrm{Zr}$, belonging to the first $s$-process peak (ls, light- $s$, at neutron magic number $N=50$ ), $\mathrm{Ba}, \mathrm{La}, \mathrm{Ce}, \mathrm{Pr}, \mathrm{Nd}, \mathrm{Sm}$, belonging to the second $s$ peak (hs, heavy-s, at $N=82$ ). Moreover, for the first time, $\mathrm{Pb}$ at the termination of the $s$-process $(N=126)$, was detected. Similar high-quality spectra (with higher $\mathrm{S} / \mathrm{N} \sim 500$ ) for other 8 barium giants has been analysed by Smiljanic et al. (2007), without lead measurement. The aim of this paper is to compare our theoretical predictions with Allen \& Barbuy (2006) and Smiljanic et al. (2007) observational samples.

In Section 2 we present the models used. In Section 3 we compare our theoretical predictions with the observations and we discuss in detail the case of a barium dwarf (HD 123585) and of a barium giant (HD 27271). 
In Section 4 we perform mass transfer calculations for some stars with known orbital period. These results allow us to check the self-consistency of the dilution factor obtained for giants (or subgiants) and to estimate the depth of the thermohaline mixing for barium dwarfs.

\section{Theoretical Models}

Our models are based on FRANEC (Frascati RaphsonNewton Evolutionary Code, Chieffi \& Straniero 1989), coupled with a post-process code that includes a full network up to bismuth. According to FRANEC, TDU starts after a limited number of TPs. TDU ceases when the mass of the envelope decreases by mass loss down to $\sim 0.5 \mathrm{M}_{\odot}$. The TDU mass per pulse reaches a maximum after about ten thermal pulses and then decreases (Straniero et al. 2003). The major neutron source in AGB stars is the ${ }^{13} \mathrm{C}(\alpha, \mathrm{n}){ }^{16} \mathrm{O}$ reaction, which releases neutrons in radiative conditions during the interpulse phase (Straniero et al. 1995). During TDU, a small amount of protons is assumed to penetrate in the top layers of the $\mathrm{He}$ intershell. At $\mathrm{H}$ reignition, protons are captured by the abundant ${ }^{12} \mathrm{C}$, via ${ }^{12} \mathrm{C}(\mathrm{p}, \gamma){ }^{13} \mathrm{~N}\left(\beta^{+} \nu\right){ }^{13} \mathrm{C}$. We use AGB models having initial masses of $1.4,1.5,2$ and $3 \mathrm{M}_{\odot}$, different metallicities and a range of ${ }^{13} \mathrm{C}$-pocket efficiencies, from $\mathrm{ST} \times 2$ down to ST/12. Case ST is the standard choice of Gallino et al. (1998), which was shown to best reproduce the solar main component of the $s$-process (Arlandini et al. 1999). The $\mathrm{ST} \times 2$ case roughly corresponds to the maximum ${ }^{13} \mathrm{C}$ pocket efficiency. Higher proton abundances would lead to the formation of ${ }^{14} \mathrm{~N}$ at expenses of ${ }^{13} \mathrm{C}$. Moreover, ${ }^{14} \mathrm{~N}$ would act as a major neutron poison. We may define a minimum ${ }^{13} \mathrm{C}$-pocket as the one that significantly affects the final $s$-process distribution. This limit corresponds roughly to ST/6 at solar metallicity and decreases with decreasing metallicity, given that the neutron exposure is inversely proportional to the iron seeds. A second minor neutron source is driven by the reaction ${ }^{22} \mathrm{Ne}(\alpha, \mathrm{n}){ }^{25} \mathrm{Mg}$, partially activated during TPs, producing a neutron burst of small neutron exposure and high peak neutron density. For a detailed description of the models we refer to Busso, Gallino \& Wasserburg (1999) and Straniero et al. (2003).

As barium stars owe their $s$ enhancement to mass transfer, the $s$-rich material from the AGB donor was mixed and diluted with the envelope of the future barium star. Defining the dilution factor as $M_{\star}^{\text {env }} / M_{\mathrm{AGB}}^{\text {transf }}=10^{d i l}$, where $M_{\star}^{\text {env }}$ is the mass of the envelope of the observed star after the mass transfer, $M_{\mathrm{AGB}}^{\text {transf }}$ is the mass transferred from the AGB, we obtain the diluted theoretical abundances ${ }^{1}$ :

$$
\left[\frac{\mathrm{X}}{\mathrm{Fe}}\right]=\log \left(10^{[\mathrm{X} / \mathrm{Fe}]^{\mathrm{ini}}} f+10^{[\mathrm{X} / \mathrm{Fe}]^{\mathrm{AGB}}-d i l}\right),
$$

where $f=1-10^{-d i l}$ and $[\mathrm{X} / \mathrm{Fe}]^{\mathrm{AGB}}$ is the abundance of the element $X$ in the AGB. We suppose that the two stars formed from the same cloud of interstellar gas.

\footnotetext{
${ }^{1}$ The standard spectroscopic notation is adopted: $[\mathrm{A} / \mathrm{B}]=$ $\log _{10}\left(N_{\mathrm{A}} / N_{\mathrm{B}}\right)_{\star}-\log _{10}\left(N_{\mathrm{A}} / N_{\mathrm{B}}\right)_{\odot}$.
}

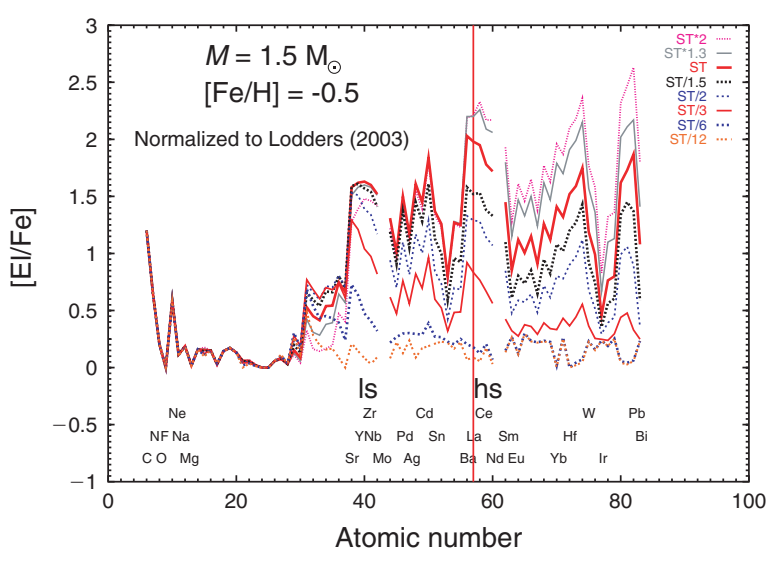

Figure 1 Theoretical predictions of [El/Fe] for AGB models of $M=1.5 \mathrm{M}_{\odot},[\mathrm{Fe} / \mathrm{H}]=-0.5$ and various ${ }^{13} \mathrm{C}$-pocket efficiencies (from ST $\times 2$ down to ST/12). We normalized to the photosperic values by Lodders (2003).

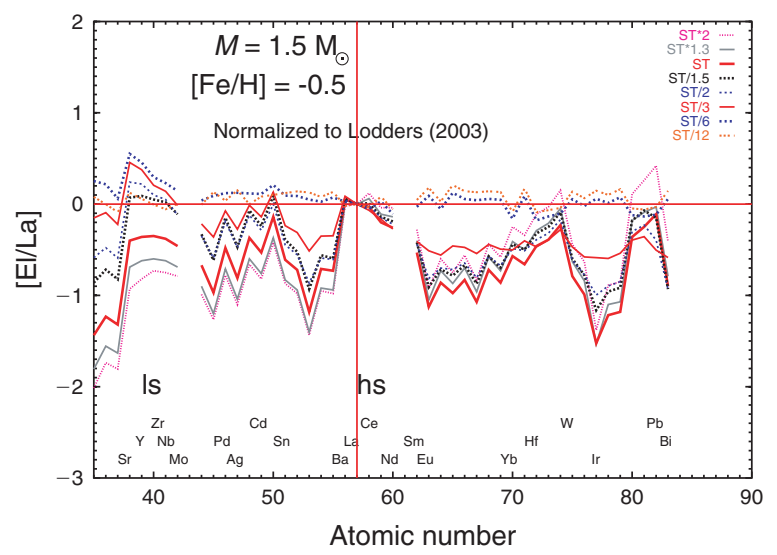

Figure 2 Theoretical predictions [El/La] for elements from $\mathrm{Sr}$ to Bi for AGB models of $M=1.5 \mathrm{M}_{\odot},[\mathrm{Fe} / \mathrm{H}]=-0.5$ and various ${ }^{13} \mathrm{C}$-pocket efficiencies (from ST $\times 2$ down to ST/12).

Dilution will lower the abundances of the elements, but will not change the shape of the $s$-process distribution. If $d i l \simeq 0$, it means that no mixing had occurred after the mass transfer, and the observed abundances are the same as in the AGB star.

We present in Figure 1 theoretical predictions for elements from carbon to bismuth using AGB models of $1.5 \mathrm{M}_{\odot}$ and $[\mathrm{Fe} / \mathrm{H}]=-0.5$ at the last TDU episode for a range of ${ }^{13} \mathrm{C}$-pocket efficiencies. Note that about half of the AGB mass ejected by stellar winds has a chemical composition shown in the Figure. Indeed, as recalled in Section 2, according to FRANEC, TDU ceases when the envelope mass is of the order of $0.5 \mathrm{M}_{\odot}$. The same results are shown in Figure 2 for heavier elements normalized to lanthanum, which is the best representative element of the hs peak.

\section{Results}

The Allen \& Barbuy (2006) sample contains both dwarfs and barium giants or subgiants. Barium dwarfs have negligible convective envelopes. In order to estimate the mass of convective envelope of our sample stars, we 


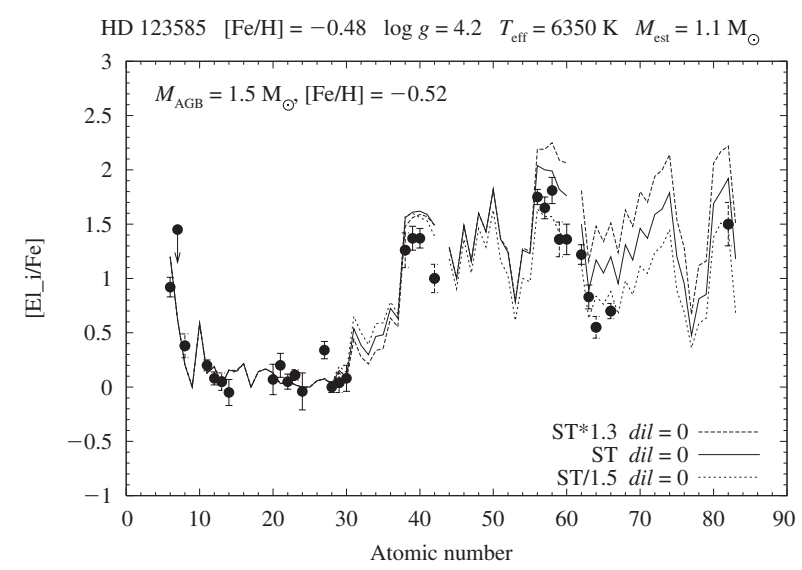

Figure 3 The Barium dwarf star HD 123585 elemental spectroscopic distribution compared with theoretical predictions. We plot the theoretical abundances without dilution, for an AGB with initial mass $M_{\mathrm{AGB}}=1.5 \mathrm{M}_{\odot},[\mathrm{Fe} / \mathrm{H}]=-0.52$ and three different ${ }^{13} \mathrm{C}$-pocket efficiencies (ST, ST $\times 1.3$ and ST/1.5).

used the Window To The Stars (WTTS) interface to Peter Eggleton's TWIN single and binary stellar evolution code (Izzard \& Glebbeek 2006). Giants and subgiants have larger convective envelopes and some of the giants have already suffered the first dredge-up (FDU), so we expect large dilution factors in these cases.

In order to find a possible solution for a barium star, we seek for the ${ }^{13} \mathrm{C}$-pocket efficiency that would reproduce the observed trend for the elements belonging to the three $s$-peaks, ls, hs and $\mathrm{Pb}$. We define

$$
[\mathrm{ls} / \mathrm{Fe}]=\frac{[\mathrm{Y} / \mathrm{Fe}]+[\mathrm{Zr} / \mathrm{Fe}]}{2}
$$

and

$$
[\mathrm{hs} / \mathrm{Fe}]=\frac{[\mathrm{La} / \mathrm{Fe}]+[\mathrm{Nd} / \mathrm{Fe}]+[\mathrm{Sm} / \mathrm{Fe}]}{3}
$$

and their ratios [hs/ls] and $[\mathrm{Pb} / \mathrm{hs}]$, two $s$-process indexes independent of the dilution factor.

In Figures 3-5, we compare spectroscopic observations of the barium dwarf HD 123585 and the barium giant HD 27271 by Allen \& Barbuy $(2006)^{2}$.

HD 123585 is a barium dwarf of metallicity $[\mathrm{Fe} / \mathrm{H}]=-0.48$ with orbital period of 458 days (Pourbaix and Jorissen 2000). The $s$ enhancement is quite large: $[\mathrm{ls} / \mathrm{Fe}] \sim 1.4 \mathrm{dex},[\mathrm{hs} / \mathrm{Fe}] \sim 1.7 \mathrm{dex}$ and $[\mathrm{hs} / \mathrm{Fe}] \sim 1.5 \mathrm{dex}$. For HD 123585, the ST case (the solid line in Figure 3) satisfies this condition: in the theoretical case $[\mathrm{hs} / \mathrm{ls}]=0.14$ and $[\mathrm{Pb} / \mathrm{hs}]=0.17$, while from the observations we have

\footnotetext{
${ }^{2}$ The error bars in the plots are quoted from Allen \& Barbuy (2006) and represent the highest value between the uncertainty due to the atmospheric model together with the stellar parameters and the line-to-line scatter for elements with more than two lines used to derive the abundances. These error bars are plotted as solid lines. For elements where the abundances were derived from only one or two lines (O, 2 lines; $\mathrm{Sr}, 1$ line for dwarfs, 2 lines for giants; $\mathrm{Pb}, 1$ line; Mo, 1 line) we have plotted with dashed line the error bar. This value has to be considered as a lower limit of the uncertainty for the abundances of these elements.
}

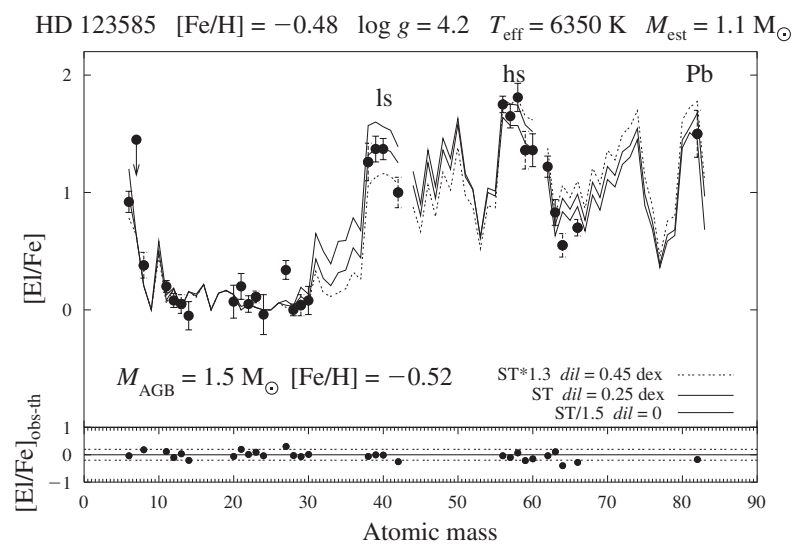

Figure 4 The Barium dwarf star HD 123585 elemental spectroscopic distribution compared with theoretical predictions. We plot the theoretical abundances with dilution, for the $1.5 \mathrm{M}_{\odot} \mathrm{AGB}$ model with $[\mathrm{Fe} / \mathrm{H}]=-0.52$ and three ${ }^{13} \mathrm{C}$-pocket efficiencies $(\mathrm{ST}, \mathrm{ST} \times 1.3$ and $\mathrm{ST} / 1.5)$.

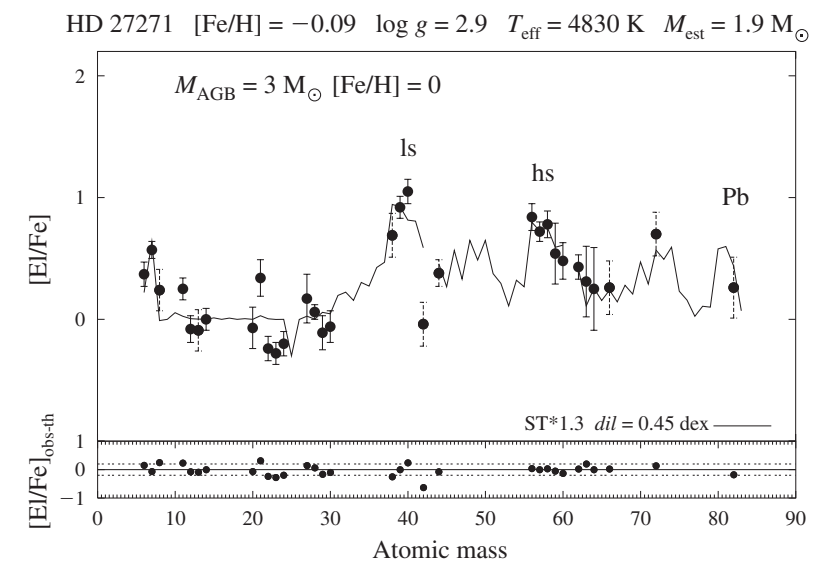

Figure 5 Barium giant HD 27271. Fit obtained with an AGB model of $3 \mathrm{M}_{\odot}$ initial mass, $[\mathrm{Fe} / \mathrm{H}]=0, \mathrm{ST} \times 1.3^{13} \mathrm{C}$-pocket efficiency and 0.45 dex dilution.

[hs/ls] and [Pb/hs] respectively 0.07 and 0.13 , with a typical uncertainty of $0.2 \mathrm{dex}$. For the other two cases plotted in Figure 3, ST $\times 1.3$ and ST/1.5, the discrepancy in $[\mathrm{hs} / \mathrm{ls}]$, with respect to the observational values, is of $\sim 0.4$ dex for the $S T \times 1.3$ case, and $\sim 0.3$ dex for the $\mathrm{ST} / 1.5$ case. The dilution factor $\mathrm{dil}$ is then chosen in order to match the $[\mathrm{hs} / \mathrm{Fe}]$ predictions with spectroscopic data. In Figure 4 we notice that, after dilution, all the three cases shown $(\mathrm{ST} \times 1.3, \mathrm{ST}$ and ST/1.5) match the hs-peak, but only the ST case matches the ls-peak.

Very similar solutions are obtained with the ST case and an initial mass of the AGB donor of 2 and $3 \mathrm{M}_{\odot}$. The dilution factor is $0.25 \mathrm{dex}$ for the $1.5-\mathrm{M}_{\odot}$ model, $0.6 \mathrm{dex}$ for the $2-\mathrm{M}_{\odot}$ model and 0.5 for the $3-\mathrm{M}_{\odot}$ model. Mo, Gd and Dy, each derived from 1 line, are lower than expected. Note that our relative $s$-process expectations of close-by elements are quite robust and are based on accurate neutron capture cross sections. 


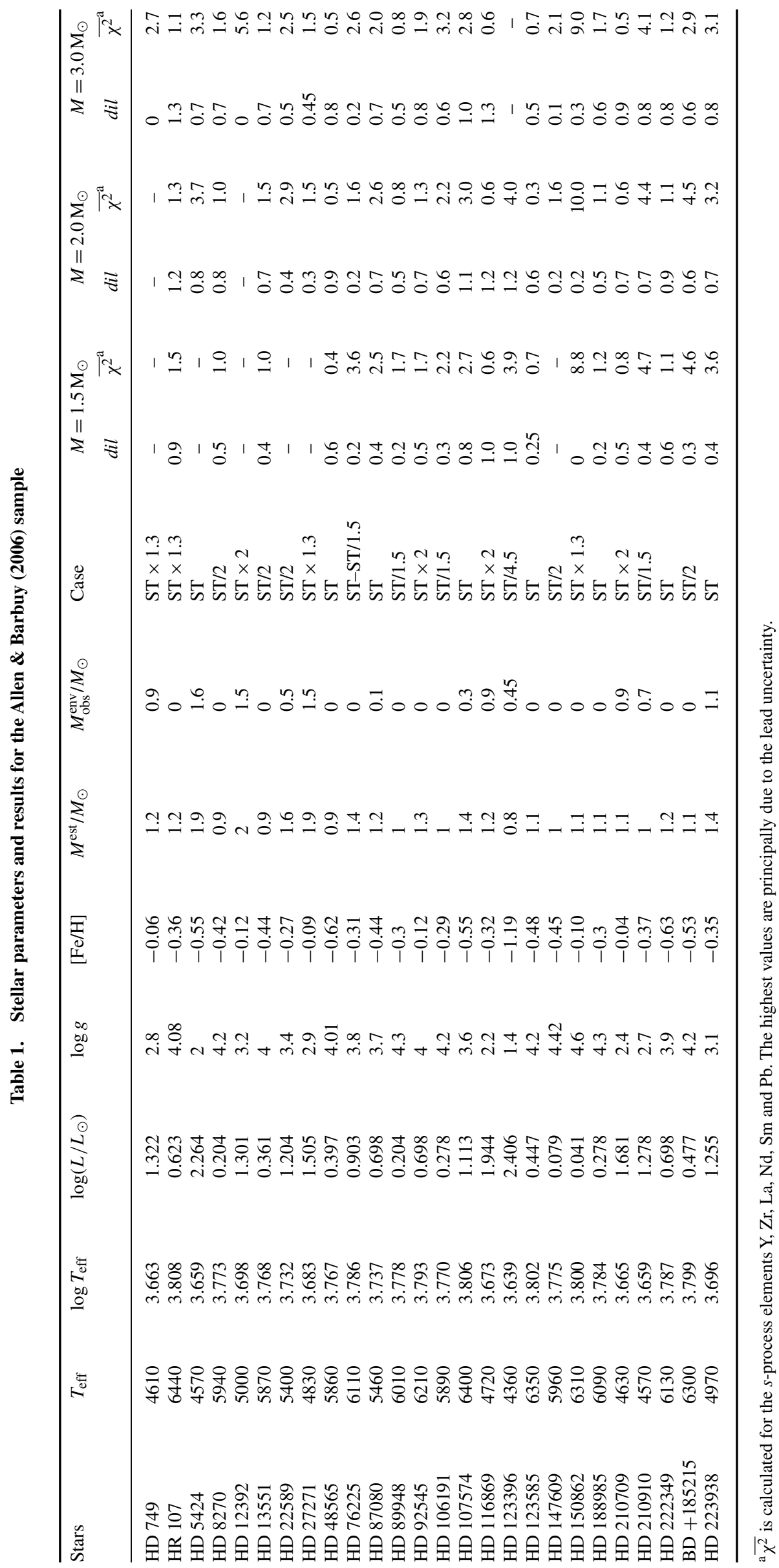




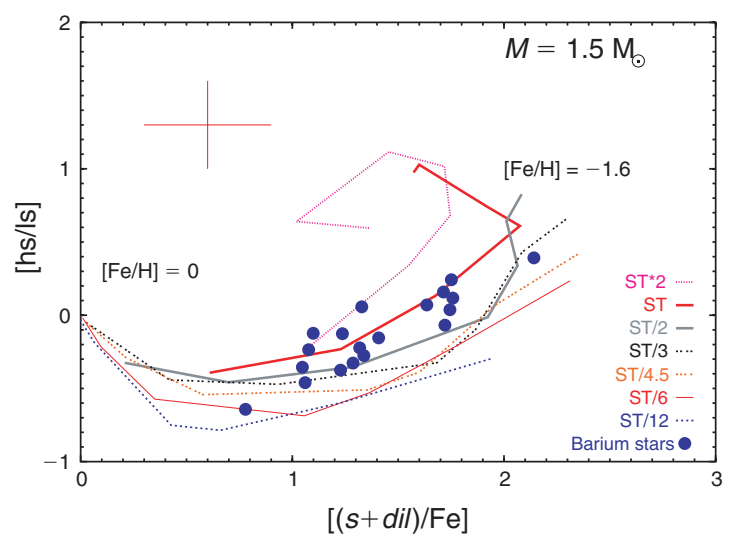

Figure 6 Theoretical results for $[\mathrm{hs} / \mathrm{ls}]$ versus $[(s+d i l) / \mathrm{Fe}]$ (where dil is the dilution factor), using AGB models of initial mass $M_{\mathrm{ini}}^{\mathrm{AGB}}=1.5 \mathrm{M}_{\odot}$ and a range of ${ }^{13} \mathrm{C}$-pocket efficiencies. Full circles correspond to spectroscopic observations of barium stars of the Allen \& Barbuy (2006) sample. For higher ${ }^{13} \mathrm{C}$-pocket efficiency and decreasing the metallicity, $[(s+d i l) / \mathrm{Fe}]$ decreases because the $s$-process feeds mostly $\mathrm{Pb}$

HD 27271 is a barium giant of solar metallicity with orbital period of 1694 days (Udry et al. 1998a). For this star $[\mathrm{ls} / \mathrm{Fe}] \sim 1,[\mathrm{hs} / \mathrm{Fe}] \sim 0.5$ and $[\mathrm{Pb} / \mathrm{Fe}] \sim 0.3 \mathrm{dex}$. We were able to reproduce the observations using a case $\mathrm{ST} \times 1.3$, and dilution factors $0.1,0.3$ and 0.45 dex for models with $M=1.5,2$ and $3 \mathrm{M}_{\odot}$, respectively. The solution found for the $1.5-\mathrm{M}_{\odot}$ initial mass of the AGB donor was discarded, because the estimated mass of this star is $M_{\text {est }}=1.9 \mathrm{M}_{\odot}$ (Allen \& Barbuy 2006). Mo, with only one line detected, appears too low.

In Table 1, the major characteristics of the Allen \& Barbuy (2006) sample are shown. For each star, we report the effective temperature, luminosity, $\log g$, the metallicity, the estimated mass of the star, the convective envelope mass according to FRANEC (see also Izzard \& Glebbeek 2006), and the parameters used to obtain the theoretical fits (efficiency of the ${ }^{13} \mathrm{C}$-pocket, initial mass of the AGB, dilution factor and reduced $\chi$-squared $\overline{\chi^{2}}$ to evaluate the goodness of the fit). Note as, in general, the adopted ${ }^{13} \mathrm{C}$-pocket does not change with the initial AGB mass (Column 9). The only exception is HD 76225, with a case ST for a model with $M=1.5 \mathrm{M}_{\odot}$ and ST/1.5 for models with $M=2$ and $3 \mathrm{M}_{\odot}$. For the two giants HD 749 and HD 12392, a dilution of about 0.5 dex can be obtained by decreasing the metallicity by about 0.2 dex.

In Figure 6 we show theoretical predictions of [hs/ls] versus $[(s+d i l) / \mathrm{Fe}]$ for AGB models of $M=1.5 \mathrm{M}_{\odot}$. Here ' $[s / \mathrm{Fe}]$ ' means $([1 \mathrm{~s} / \mathrm{Fe}]+[\mathrm{hs} / \mathrm{Fe}]) / 2$ and 'dil' is the dilution factor used in the model. Stars interpreted with a $M_{\mathrm{ini}}^{\mathrm{AGB}}=1.5 \mathrm{M}_{\odot}$ are represented with full circles (see Table 1, Columns 10 and 11). Typical error bars are shown. Similarly, in Figure 7, we shown theoretical predictions as compared with observations of $[\mathrm{Pb} / \mathrm{hs}$ ] versus [hs/ls]. $[\mathrm{Pb} / \mathrm{hs}]$ shows quite a large scatter with respect to predictions, due to the fact that only one line of $\mathrm{Pb}$ has been detected.

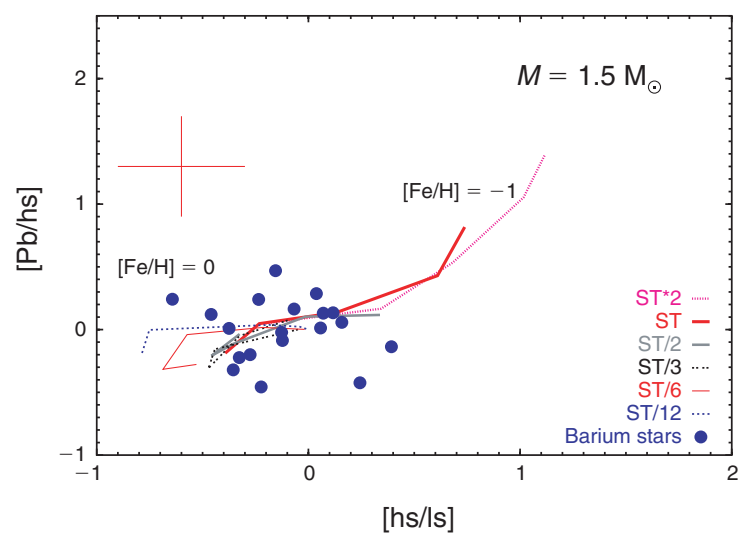

Figure 7 The same as Figure 6 , but for [Pb/hs] versus [hs/ls].

For more detailed descriptions on the other stars we refer to Husti $\mathrm{PhD}$ Thesis (2008), and to the preliminary analyses by Husti \& Gallino (2008) and Husti, Gallino \& Straniero (2008).

Smiljanic et al. (2007) analysed a sample of 8 classical and mild barium giants, known in the literature, reanalysed at high resolution and $\mathrm{S} / \mathrm{N}$. The estimated mass is in the range 1.9 to $4.2 \mathrm{M}_{\odot}$ and the metallicity ranges from solar down to $[\mathrm{Fe} / \mathrm{H}] \sim-0.3$. For most of these giants, only one line was detected for $\mathrm{Sr}$ and $\mathrm{Sm}$, and two lines for Nd. For these elements no error bars are given by the authors. Y, Zr, $\mathrm{La}$ and $\mathrm{Ce}$ are better determined. The quoted error bars are typically higher in the Smiljanic et al. (2007) sample than in Allen \& Barbuy (2006), despite higher S/N spectra. No lead abundances were determined. A preliminary analysis of the two barium stars HD 116713 and HD 205011 has been presented by Husti \& Gallino (2008). In Table 3, we show the main characteristic of this sample, with their theoretical interpretations, in particular, our $[\mathrm{Pb} / \mathrm{Fe}]$ predictions. For the two stars HD 46407 and HD 204075, a dilution of $0.7 \mathrm{dex}$ and $[\mathrm{Pb} / \mathrm{Fe}] \sim 1.4$ would be obtained by decreasing the metallicity by $\sim 0.2$ dex. All stars have a typical [hs/ls] close to 0 . The only exception is HD 139195 for which $[\mathrm{hs} / \mathrm{ls}] \sim 0.25$.

\section{Mass-Transfer Calculations}

Boffin \& Jorissen (1988) showed that the amount of mass transferred from an AGB star to its companion through stellar winds $\left(\Delta M_{2}\right)$ depends on the present value of the orbital separation, the mass of the barium star and of the white dwarf companion:

$$
\begin{aligned}
\Delta M_{2}= & \frac{1}{\sqrt{1-e^{2}}}\left(\frac{G M_{2}}{V_{\text {wind }}^{2}}\right)^{2} \frac{\alpha}{2 A} \Delta M_{1} \\
& \times\left[\frac{1}{1+G\left(M_{1}+M_{2}\right) / A V_{\text {wind }}^{2}}\right]^{3 / 2},
\end{aligned}
$$

where $M_{1}$ is the mass of the white dwarf, $M_{2}$ the mass of the barium star, $\Delta M_{1}$ is the mass lost by winds by 
Table 2. [ls/Fe], [hs/Fe], $[\mathrm{Pb} / \mathrm{Fe}]$ and their ratios $[\mathrm{hs} / \mathrm{ls}]$ and $[\mathrm{Pb} / \mathrm{hs}]$ for the Allen \& Barbuy (2006) sample

\begin{tabular}{lrrrrrr}
\hline Stars & {$[\mathrm{Fe} / \mathrm{H}]$} & {$[\mathrm{ls} / \mathrm{Fe}]$} & {$[\mathrm{hs} / \mathrm{Fe}]$} & {$[\mathrm{Pb} / \mathrm{Fe}]$} & {$[\mathrm{hs} / \mathrm{ls}]$} & {$[\mathrm{Pb} / \mathrm{hs}]$} \\
\hline HD 749 & -0.06 & 1.32 & 1.17 & 0.38 & -0.14 & -0.79 \\
HR 107 & -0.36 & 0.59 & 0.43 & 0.90 & -0.16 & 0.47 \\
HD 5424 & -0.55 & 1.19 & 1.51 & 1.10 & 0.32 & -0.41 \\
HD 8270 & -0.42 & 0.98 & 0.70 & 0.50 & -0.28 & -0.20 \\
HD 12392 & -0.12 & 1.29 & 1.52 & 1.15 & 0.24 & -0.37 \\
HD 13551 & -0.44 & 1.05 & 0.72 & 0.50 & -0.33 & -0.22 \\
HD 22589 & -0.27 & 0.95 & 0.36 & -0.15 & -0.59 & -0.51 \\
HD 27271 & -0.09 & 0.97 & 0.55 & 0.31 & -0.41 & -0.24 \\
HD 48565 & -0.62 & 1.10 & 1.22 & 1.35 & 0.12 & 0.13 \\
HD 76225 & -0.31 & 1.22 & 0.84 & 0.85 & -0.38 & 0.01 \\
HD 87080 & -0.44 & 1.23 & 1.47 & 1.05 & 0.24 & -0.42 \\
HD 89948 & -0.30 & 1.03 & 0.67 & 0.35 & -0.36 & -0.32 \\
HD 92545 & -0.12 & 0.70 & 0.46 & 0.70 & -0.24 & 0.24 \\
HD 106191 & -0.29 & 0.99 & 0.53 & 0.65 & -0.46 & 0.12 \\
HD 107574 & -0.55 & 0.96 & 0.89 & 1.05 & -0.07 & 0.16 \\
HD 116869 & -0.32 & 0.64 & 0.79 & 0.85 & 0.16 & 0.06 \\
HD 123396 & -1.19 & 0.95 & 1.34 & 1.20 & 0.39 & -0.14 \\
HD 123585 & -0.48 & 1.35 & 1.42 & 1.55 & 0.07 & 0.13 \\
HD 147609 & -0.45 & 1.57 & 1.35 & 0.78 & -0.22 & -0.57 \\
HD 150862 & -0.10 & 1.10 & 0.46 & 0.70 & -0.64 & 0.24 \\
HD 188985 & -0.30 & 1.10 & 0.97 & 0.95 & -0.13 & -0.02 \\
HD 210709 & -0.04 & 0.66 & 0.54 & 0.45 & -0.12 & -0.09 \\
HD 210910 & -0.37 & 0.45 & 0.57 & -0.04 & 0.12 & -0.61 \\
HD 222349 & -0.63 & 1.13 & 1.16 & 0.45 & 0.04 \\
BD + 185215 & -0.53 & 1.13 & 0.91 & 0.97 & -0.22 & -0.46 \\
HD 223938 & -0.35 & 0.90 & 0.96 & & 0.06 & 0.01 \\
\hline & & & & & \\
\hline
\end{tabular}

Table 3. Stellar parameters and results for the Smiljanic et al. (2007) sample

\begin{tabular}{|c|c|c|c|c|c|c|c|c|}
\hline Stars & {$[\mathrm{Fe} / \mathrm{H}]$} & $T_{\text {eff }}$ & $\log g$ & $M_{\text {est }}$ & Case & $\begin{array}{c}M=3 \mathrm{M}_{\odot} \\
\text { dil }\end{array}$ & $\begin{array}{c}M=4 \mathrm{M}_{\odot} \\
\text { dil }\end{array}$ & {$[\mathrm{Pb} / \mathrm{Fe}]_{\mathrm{th}}$} \\
\hline HD 46407 & -0.09 & 4940 & 2.65 & 2.3 & $\mathrm{ST} \times 2$ & 0.2 & - & 1.1 \\
\hline HD 104979 & -0.35 & 4920 & 2.58 & 2.3 & $\mathrm{ST} \times 1.3$ & 1.4 & - & 0.6 \\
\hline HD 116713 & -0.12 & 4790 & 2.67 & 1.9 & $\mathrm{ST} \times 2$ & 0.5 & - & 0.8 \\
\hline HD 139195 & -0.04 & 4910 & 2.41 & 2.4 & ST & 0.8 & - & 0.0 \\
\hline HD 181053 & -0.35 & 4920 & 2.58 & 2.2 & ST & 1.7 & - & 0.2 \\
\hline HD 202109 & -0.04 & 4910 & 2.41 & 3.0 & $\mathrm{ST} \times 2$ & 1.5 & - & 0.2 \\
\hline HD 204075 & -0.09 & 5250 & 2.49 & 4.2 & $\mathrm{ST} \times 2$ & - & 0.3 & 1.2 \\
\hline HD 205011 & -0.14 & 4780 & 2.41 & 2.2 & $\mathrm{ST} \times 1.3$ & 0.6 & - & 0.4 \\
\hline
\end{tabular}

the AGB companion, $A$ is the semi-major axis, $\alpha$ is a free parameter put equal to 2 (Boffin \& Jorissen 1988) and $e$ the eccentricity. The semi-major axis $A$ varies with the mass exchange between the two binaries. A choice $A=$ constant may be considered an acceptable approximation during the superwind phase at the end of the AGB. Our FRANEC models adopt the Reimers (1977) mass-loss formula and do not explicitly include superwinds, however we recall that almost $0.5 \mathrm{M}_{\odot}$ of the envelope mass is ejected with the same $s$-process composition.

Thus knowing the mass of the convective envelope from FRANEC and the dilution factor obtained from the abundance fits we may derive the velocity of the wind, for the giants and subgiants, of which we know the orbital period, by comparing $\Delta M_{2}$ from the equation above with $M_{\mathrm{AGB}}^{\text {transf }}$ from Section 2.

In Figure 8 we plot $\Delta M_{2}\left(\equiv M_{\mathrm{AGB}}^{\text {transf }}\right)$ as a function of the wind velocity and the value of $M_{\mathrm{AGB}}^{\text {transf }}\left(\equiv \Delta M_{2}\right.$ ) obtained from the dilution factor used to get the fit. For HD 27271 we obtain 2 solutions: $V_{\text {wind }} \simeq 23 \mathrm{~km} \mathrm{~s}^{-1}$ if we consider the initial mass of the AGB to be $3 \mathrm{M}_{\odot}$, and $V_{\text {wind }} \simeq 18 \mathrm{~km} \mathrm{~s}^{-1}$ if we consider the initial mass of the AGB to be $2 \mathrm{M}_{\odot}$. The wind velocity for AGB stars was estimated to be in the interval 200-40 $\mathrm{km} \mathrm{s}^{-1}$ (Pottasch 1984; Knapp 1985). Only the solution obtained for the $3-\mathrm{M}_{\odot}$ case is in this range.

Half of the sample are barium dwarfs. The range of estimated mass of dwarfs by Allen \& Barbuy (2006) is 


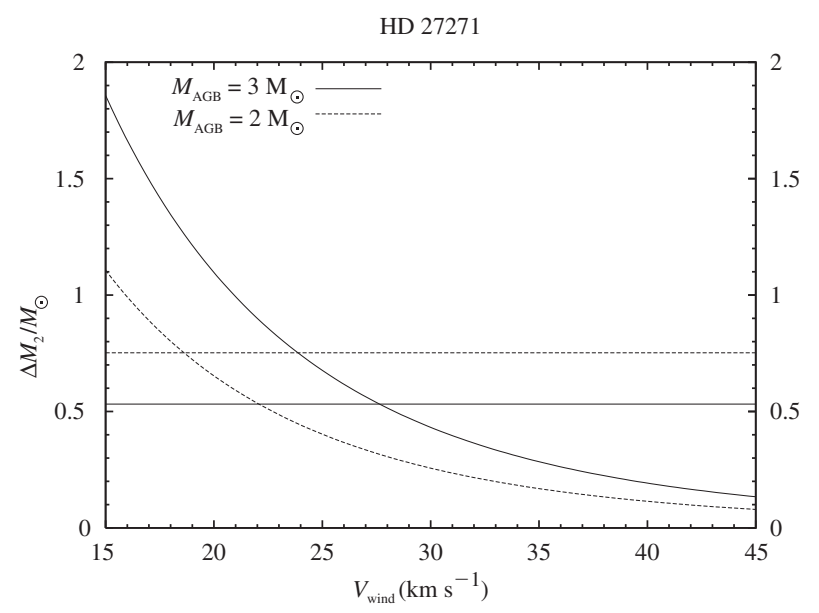

Figure 8 Transferred mass onto the barium giant HD 27271 versus wind velocity.

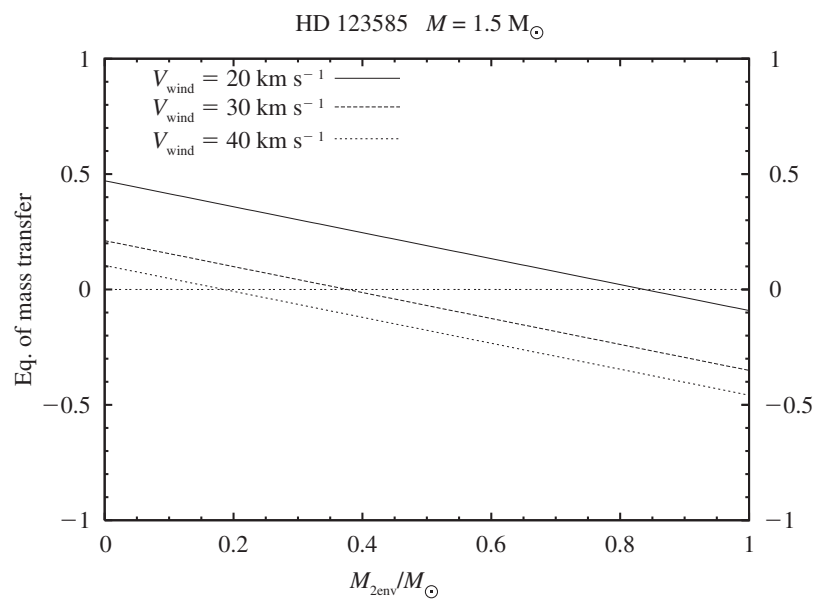

Figure 9 Equation of the mass transfer versus mass of the envelope mixed with the $s$-enhanced material, for star HD 123585, the case of the $1.5-\mathrm{M}_{\odot}$ initial AGB mass of the companion.

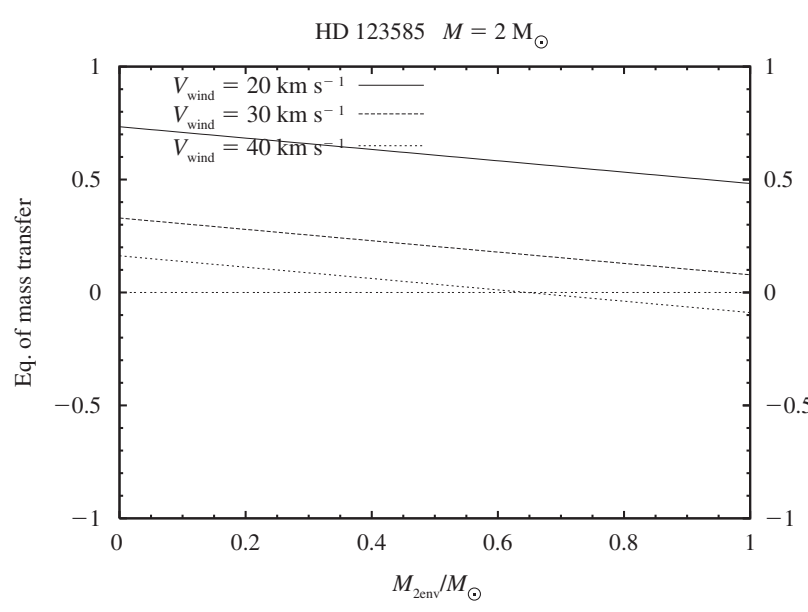

Figure 10 Equation of the mass transfer versus mass of the envelope mixed with the $s$-enhanced material, for star HD 123585, the case of the $2-\mathrm{M}_{\odot}$ initial AGB mass of the companion.

between $0.9 \leq M_{\mathrm{est}} / M_{\odot} \leq 1.4$. No convective envelope is expected for these objects. Many of the stars need dilution factor higher than $0.5 \mathrm{dex}$. This might be explained by moderate to strong thermohaline mixing. Putting to

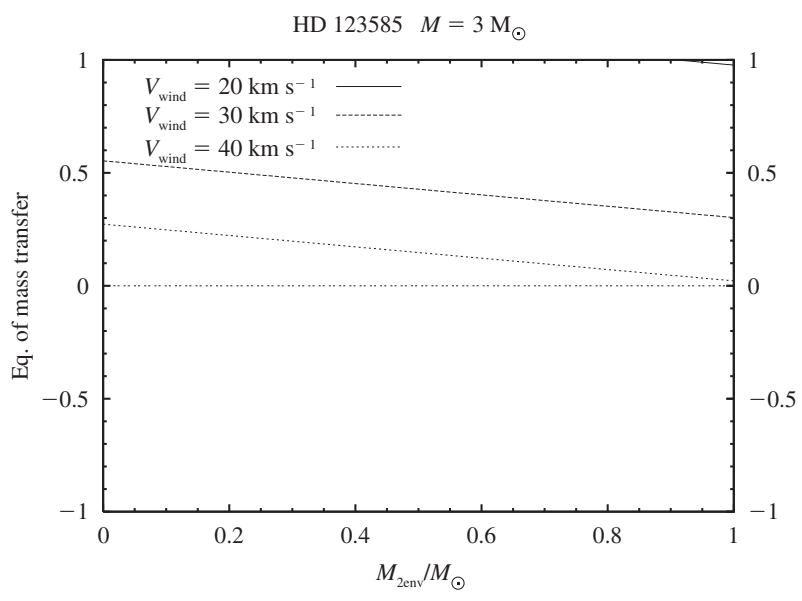

Figure 11 Equation of the mass transfer versus mass of the envelope mixed with the $s$-enhanced material, for star HD 123585, the case of the $3-\mathrm{M}_{\odot}$ initial AGB mass of the companion.

zero the mass transfer equation, and replacing $\Delta M_{2}$ with $M_{\star}^{\text {env }} / 10^{d i l}$, we may estimate the depth of the mixing. In Figures 9, 10 and 11, we have plotted the equation of mass transfer versus the amount of envelope mass over which the $s$-rich material is diluted due to thermohaline mixing, for HD 123585. In the cases with 2 and $3 \mathrm{M}_{\odot}$, the depth of the mixing (in mass) would exceed the mass of the barium star for $V_{\text {wind }}=20 \mathrm{~km} \mathrm{~s}^{-1}$, so we discard these cases. For $1.5 \mathrm{M}_{\odot}$, the amount of envelope mass mixed with the $s$-rich transferred material is in the range $\sim 0.20$ to $\sim 0.85 \mathrm{M}_{\odot}$.

In Table 4 we report the period and the eccentricity available in the literature for six of the barium stars of our sample (HD 5424, HD 22589, HD 27271: Udry et al. 1998a,b; HD 89948, HD 107574, HD 123585: Pourbaix \& Jorissen 2000), as well as the calculated wind velocity for giants (or subgiants), having various masses of the AGB donor. For HD 5424 we obtain two solutions in the range 20-40 $\mathrm{km} \mathrm{s}^{-1}$, for HD 25589 and HD 27271 we have only one solution in the given range, while for HD 107574 we have no solution in the chosen interval, the closest one is for a $1.5-\mathrm{M}_{\odot}$ AGB companion, with $V_{\text {wind }}=42 \mathrm{~km} \mathrm{~s}^{-1}$.

In Tables 5 and 6 we present the depth in mass of the thermohaline mixing for the two dwarf stars of the sample with known orbital period. The depth of the mixing depends on the mass of the AGB and on the assumed wind velocity. For HD 89948 we obtain similar results as for HD 123585. The mixing seems to be deeper for the lower metallicity star.

\section{Conclusions}

We have found theoretical interpretations for the abundance patterns of 26 barium dwarfs, barium subgiants and giants from Allen \& Barbuy (2006) and of 8 barium giants from Smiljanic et al. (2007). In order to obtain these solutions, we have applied various dilution factors (from 0 to $1.5 \mathrm{dex}$ ) due to the mixing of the $s$-enhanced material with the envelope of the accretor. In most cases, we have not found unique solutions: models having the same 
Table 4. Parameters of the theoretical solutions and calculated wind velocities for giants and subgiants

\begin{tabular}{lrcccc}
\hline Stars & $P$ (days) & $e$ & $\begin{array}{c}M_{\mathrm{AGB}}=3 \mathrm{M}_{\odot} \\
V_{\text {wind }} \\
\left(\mathrm{km} \mathrm{s}^{-1}\right)\end{array}$ & $\begin{array}{c}M_{\mathrm{AGB}}=2 \mathrm{M}_{\odot} \\
V_{\text {wind }} \\
\left(\mathrm{km} \mathrm{s}^{-1}\right)\end{array}$ & $\begin{array}{c}M_{\mathrm{AGB}}=1.5 \mathrm{M}_{\odot} \\
V_{\text {wind }} \\
\left(\mathrm{km} \mathrm{s}^{-1}\right)\end{array}$ \\
\hline HD 5424 & 1881 & 0.225 & 32 & - & - \\
HD 22589 & 5721 & 0.240 & 22 & 17 & - \\
HD 27271 & 1694 & 0.217 & 23 & 18 & - \\
HD 89948 & 668 & 0.117 & - & - & - \\
HD 107574 & 1350 & 0.081 & $>45$ & $>45$ & 42 \\
HD 123585 & 458 & 0.062 & - & - & - \\
\hline
\end{tabular}

Table 5. HD 89948, depth of thermohaline mixing

\begin{tabular}{lccc}
\hline $\mathrm{HD} 89948$ & $M_{\mathrm{AGB}}=1.5 \mathrm{M}_{\odot}$ & $M_{\mathrm{AGB}}=2 \mathrm{M}_{\odot}$ & $M_{\mathrm{AGB}}=3 \mathrm{M}_{\odot}$ \\
\hline$V_{\text {wind }}=20 \mathrm{~km} \mathrm{~s}^{-1}$ & 0.5 & $>1$ & $>1$ \\
$V_{\text {wind }}=30 \mathrm{~km} \mathrm{~s}^{-1}$ & 0.22 & 0.7 & $>1$ \\
$V_{\text {wind }}=40 \mathrm{~km} \mathrm{~s}^{-1}$ & 0.10 & 0.3 & 0.55 \\
\hline
\end{tabular}

Table 6. HD 123585, depth of thermohaline mixing

\begin{tabular}{lccc}
\hline $\mathrm{HD} 123585$ & $M_{\mathrm{AGB}}=1.5 \mathrm{M}_{\odot}$ & $M_{\mathrm{AGB}}=2 \mathrm{M}_{\odot}$ & $M_{\mathrm{AGB}}=3 \mathrm{M}_{\odot}$ \\
\hline$V_{\text {wind }}=20 \mathrm{~km} \mathrm{~s}^{-1}$ & 0.83 & $>1$ & $>1$ \\
$V_{\text {wind }}=30 \mathrm{~km} \mathrm{~s}^{-1}$ & 0.37 & $>1$ & $>1$ \\
$V_{\text {wind }}=40 \mathrm{~km} \mathrm{~s}^{-1}$ & 0.18 & 0.65 & $>1$ \\
\hline
\end{tabular}

metallicity and ${ }^{13} \mathrm{C}$-pocket efficiency, but different masses and dilution factors match just as well the observations.

For barium giants and subgiants that have large convective envelopes, an appreciable dilution factor has to be expected, as confirmed by our results. For several barium dwarfs that do not have a convective envelope, an important dilution factor must also be applied. This comes as a proof that efficient thermohaline mixing is active in these stars (Stancliffe et al. 2007).

We have performed some mass transfer calculations for the stars that have the orbital period reported in literature. For barium giants and subgiants we could thus obtain the stellar wind velocity, while for barium dwarfs, assuming a certain initial mass of the AGB and a certain value of the wind velocity, we could estimate the depth of the thermohaline mixing.

Further observations of barium stars with high S/N and spectral resolution would be very helpful.

\section{Acknowledgments}

We thank the referee Rob Izzard for his incisive suggestions to improve the paper. Work supported by the Italian MIUR-PRIN 2006 Project 'Late Phases of Stellar Evolution: Nucleosynthesis in Supernovae, AGB Stars, Planetary Nebulae'.

\section{References}

Allen, D. M. \& Barbuy, B., 2006, A\&A, 454, 895

Arlandini, C., Käppeler, F., Wisshak, K., Gallino, R., Lugaro, M., Busso, M. \& Straniero, O., 1999, ApJ, 525, 886
Bidelman, W. P. \& Keenan, P. C., 1951, ApJ, 114, 473

Boffin, H. M. J. \& Jorissen, A., 1988, A\&A, 205, 155

Busso, M., Lambert, D. L., Beglio, L., Gallino, R., Raiteri, C. M. \& Smith, V. V., 1995, ApJ, 446, 775

Busso, M., Gallino, R. \& Wasserburg, G. J., 1999, ARAA, 37, 239

Busso, M., Gallino, R., Lambert, D. L., Travaglio, C. \& Smith, V. V., 2001, ApJ, 557, 802

Chieffi, A. \& Straniero, O., 1989, ApJS, 71, 47

Gallino, R. et al., 1998, ApJ, 497, 388

Husti, L., 2008, PhD Thesis, Turin University

Husti, L. \& Gallino, R., 2008, AIPC, 1001, 139

Husti, L., Gallino, R. \& Straniero, O., 2008, AIPC, 990, 161

Knapp, R., 1985, ApJ, 293, 273

Izzard, R. G. \& Glebbeek, E., 2006, NewA, 12, 161

Lodders, K., 2003, ApJ, 591, 1220

Jorissen, A. \& Mayor, M., 1988, A\&A, 198, 187

Pottasch, S. R., 1984, ASSL, 107

Pourbaix, D. \& Jorissen, A., 2000, A\&AS, 145, 161

Reimers, D., 1977, A\&A, 61, 217

Smiljanic, R., Porto de Mello, G. F. \& da Silva, L., 2007, A\&A, 468, 679

Stancliffe, R. J., Glebbeek, E., Izzard, R. G. \& Pols, O. R., 2007, A\&A, 464, 57

Straniero, O. et al., 1995, ApJ, 440, 85

Straniero, O., Domínguez, I., Cristallo, S. \& Gallino, R., 2003, PASA, 20, 389

Udry, S. et al., 1998a, A\&AS, 131, 25

Udry, S. et al., 1998b, A\&AS, 131, 43 